\title{
Activities of daily living in people with lower limb amputation: outcomes of an intervention to reduce dependence in pre- prosthetic phase
}

\author{
Iván De-Rosende Celeiro ${ }^{a}$, Luisa Simón Sanjuán ${ }^{\mathrm{a}, \mathrm{b}}$ and Sergio Santos-del-Riego ${ }^{\mathrm{c}}$ \\ ${ }^{a}$ Departamento de Ciencias da Saúde, Universidade da Coruña, A Coruña, Spain; ${ }^{b}$ Servicio de Rehabilitación, \\ Complexo Hospitalario Universitario A Coruña, A Coruña, Spain; ${ }^{c}$ Departmento de Medicina, Universidade da \\ Coruña, A Coruña, Spain
}

\begin{abstract}
Purpose: The main objective was to assess the change in the functional independence in basic activities of daily living (ADL) following a pre-prosthetic intervention in people with lower-limb amputation (LLA). Secondary objectives were to identify the factors contributing to the success of this intervention, and to analyze the effects on the presence of unmet needs for home adaptation.

Method: The ADL intervention was early and pre-prosthetic; it was focused on six self-care activities. Fifty-two adults with LLA, who required assistance in self-care, were included. Functional independence (Barthel) was assessed at baseline and after intervention (T2). Successful intervention was defined as independent performance of all self-care activities.

Results: There was a significant improvement in Barthel scores between baseline and T2 in toileting $(p<0.001)$, bedchair transfers $(p<0.001)$, dressing $(p<0.001)$, bathing/showering $(p<0.001)$, and feeding $(p=0.025)$. The proportion of homes with an unmet need for adaptation decreased significantly in bathroom $(p=0.008)$ and other internal areas $(p=0.031)$. Intervention was successful for $61.5 \%$ of participants. In a multivariate model, age was significantly associated with successful intervention (OR 0.66, 95\%CI 0.52-0.83).

Conclusions: A short and pre-prosthetic ADL intervention improves functional independence and reduces the need for home adaptation. ADL programs should be included in rehabilitation strategies.
\end{abstract}

\section{Implications for Rehabilitation}

- $\quad$ Because basic activities of daily living (ADL) can be seriously compromised after a lower-limb amputation, it is important for this population to improve or maintain their level of independence.

- A short and pre-prosthetic ADL intervention is an effective method for an early recovery of functional independence in self-care activities and promotes home adaptation.

- Age is an important determinant of functional recovery, and most subjects can achieve independence in basic ADL regardless of the level of amputation.

- A pre-prosthetic ADL program should be included in rehabilitation strategies for adults with lower-limb amputation.

Keywords: Activities of daily living, amputation, lower limb, occupational therapy, outcomes 


\section{Introduction}

Lower-limb amputations are a common cause of disability. The lower-limb amputation incidence rates will increase substantially in the next decades due to ageing of the population and progressive increase in the incidence of diabetes mellitus and peripheral vascular disease are the major causes of amputation in Western countries.[1-5] The age of this population is growing and, consequently, amputation is often associated with comorbidity and frailty. This health condition has a profound impact on daily functioning: pain, depression, changes in body image and mobility limitations are common problems.[6-9] Moreover, the mortality rates are high; in several studies, more than one-third of the participants died during the first year after amputation.[10-13] Therefore, the promotion of autonomy and quality of life in this population group constitutes a major challenge for health policy.

Rehabilitation is a key strategy for this population,[14-18] and its main objective is to regain the highest possible level of function.[16,17] Through a holistic and multidisciplinary approach, $[15,18]$ the rehabilitation process includes several stages of varying duration: preoperative phase; pre-prosthetic stage; and, if possible, prosthetic training.[16-18] The pre-prosthetic rehabilitation should be initiated as soon as possible and is mainly aimed at range of motion and strength, residual-limb shaping, nonprosthetic mobility skills (transfers and wheelchair mobility), independence in basic activities of daily living (ADL), and training with early walking aids.[14-16,18] The primary objective of the pre-prosthetic occupational therapy intervention is to facilitate independence in self-care activities, as early as possible, through training of skills for daily functioning and advice on adaptations and technology.[6,18-21] Regarding the prosthetic phase, the selection of candidates for prosthetic rehabilitation is usually a function of the rehabilitation team with patient involvement in the decision-making process, considering pre-amputation lifestyle, clinical status, skills, and expectations concerning each person.[16,18,20] Previous research has shown that a significant proportion is unable to achieve prosthetic ambulation or abandons the use of prosthesis later.[2,22-28] The rate of prosthetic use has exceeded 50\% in studies involving people admitted for rehabilitation.[22,23,27,28] However, this rate was lower in the group of older people. A systematic review concluded that the proportion of older people who reached household mobility with prosthesis was less than half of this population.[25] In unselected geriatric patients, a population-based study found that the rate of successful prosthetic fitting was only $36 \%$.[2].

Regarding the rehabilitation outcomes after a lower-limb amputation, the majority of previous studies have focused on mobility skills, mainly on walking and prosthesis fit;[22,24,25,27,29] other studies have shown that rehabilitation reduces the presence of depression [30,31] and improves survival.[32] All these previous studies have described the outcomes obtained at the end of the rehabilitation process. However, little is known about the effects of rehabilitation interventions on independence in basic ADL.[12,33-36] Moreover, in contrast to the large number of studies on factors associated with post-rehabilitation recovery of ambulation and prosthetic use, there is little evidence regarding the determinants of ADL outcomes after a rehabilitation program.[34,36] In people with lower-limb amputation, independence in self-care activities is significantly associated with higher rates of survival after six months of rehabilitation [37] and prosthetic use,[38] and predicts a good walking ability.[39] Dependence is a major concern for lower-limb amputees; autonomy in self-care is one of the most important goals for this population at admission for rehabilitation, and dissatisfaction with these activities is common.[40] Furthermore, although the literature has highlighted the importance of regaining independence as soon as possible after an amputation, [16,18,19,21] outcomes of an intervention at the pre-prosthetic phase on selfcare activities are not known. A better understanding of the effectiveness of ADL interventions is needed to promote an evidence-based practice. Therefore, the main objective of this study was to assess changes in functional independence in self-care activities following a pre-prosthetic intervention. This intervention was considered successful when the participant achieved the independent performance of these activities. The secondary objectives were to identify factors that contribute independently to the success of this ADL intervention, and to analyze the effects of this intervention on the presence of unmet needs for home adaptation. 


\section{Methods}

\section{Study design}

This was a prospective, observational study examining changes in functional independence in selfcare activities among participants with lower-limb amputation who completed a pre-prosthetic ADL intervention.

\section{Participants}

The study was carried out in the rehabilitation service of the university hospital of A Coruña (Spain). This hospital covers a population of $\sim 550000$ individuals in northwestern Spain. The service provides multidisciplinary rehabilitation for inpatient and outpatient adults, through a team of rehabilitation physicians, physiotherapists, and occupational therapists. In people with lower-limb amputation, referral to occupational therapy is performed by a rehabilitation physician after assessing compliance with the following criteria: (a) medically stable individuals, (b) without major wound problems, (c) cognitive skills to follow simple commands, and (d) potential to improve functional independence in ADL, according to the physician's clinical judgement.

All those with lower-limb amputation consecutively admitted to the occupational therapy ward from January 2010 to April 2011 were invited to participate in the study. Inclusion criteria were: (a) individuals aged $\geq 18$ years, (b) with a major lower-limb amputation (unilateral proximal to the ankle joint or bilateral), (c) at pre-prosthetic phase, (d) medically stable, (e) without drug and alcohol problems, and (f) requiring assistance of another person to perform at least one of the six self-care activities analyzed in this study: bed-chair transfers, bathing and showering, dressing, toileting, feeding and grooming. During the study period, 59 individuals with lower-limb amputation were admitted to occupational therapy. Four subjects were not at pre-prosthetic phase. Fifty-five met the inclusion criteria, and all gave their informed consent. Three individuals did not complete the ADL intervention due to worsening of health. Therefore, 52 individuals with a mean age of 65.5 years (range 33-88, SD 11.8) were included in this study.

Ethical approval for this study was obtained from the regional ethics committee. The study followed the tenets of the Declaration of Helsinki. Patients received written and oral information about the research and signed an informed consent form before entering the study.

\section{Intervention}

The intervention analyzed in this study aimed to recover the highest possible functional independence in self-care activities. This ADL intervention was characterized by being early and short. It was carried out during the pre-prosthetic phase and completed before the patient received prosthesis. The ADL intervention sessions were individual, five times a week, lasting $\sim 45 \mathrm{~min}$ each session. An occupational therapist of the multidisciplinary team with extensive experience in the field of orthopedics conducted this intervention. Participants were involved in the decision-making process. The expected duration is $\sim 10$ sessions, according to previous clinical experience in this rehabilitation service.

The ADL intervention focused on the following activities defined in the Barthel Index (BI):[41] bedchair transfers, bathing and showering, dressing, toileting, feeding and grooming. In the current study, a successful intervention was defined as the independent performance of all six of these activities (without supervision or physical assistance), according to the BI. Before the beginning of the intervention, an assessment of functional abilities and individual needs was conducted; regarding ADL and desired lifestyle, participants established their priorities and interests. Table 1 provides an overview of the major components. A training of ADL skills was conducted under supervision of the occupational therapist. In addition, on the basis of the individual needs of each participant, the occupational therapist assessed the needs for home adaptation to promote security and independence in ADL; home adaptations included 
assistive devices and removal of architectural barriers. The occupational therapist provided advice to participants and caregivers about adaptations, costs, and installation procedure. In the occupational therapy ward, participants were trained with the necessary adaptations. The criteria leading to cessation of this intervention were: (a) success of the ADL intervention as described in this paragraph; (b) lack of potential for improvement in the activities addressed by this intervention, according to the physician's clinical judgment; (c) major adverse medical events or (d) the patient's decision to end the intervention

Table 1. Overview of the major components of the pre-prosthetic ADL intervention.

\begin{tabular}{|c|c|c|c|}
\hline Pre-functional skills training & ADL skills training & Safety education & Home adaptation \\
\hline Balance and body posture control & Transferring & Fall prevention & Removal of architectural barriers \\
\hline Positioning & Activity adaptation & Risk assessment & Home assessment \\
\hline Bed mobility & Practical skills training & Education & Advice on adaptations \\
\hline Balance training & Bed & Practical skills training & Selection and aims \\
\hline Sitting balance & Chair & Caregiver counselling & Costs \\
\hline Sitting to standing & Toilet & & Installation procedure \\
\hline \multirow[t]{3}{*}{ Standing balance } & Tub and shower & & Practical training \\
\hline & Car & & Caregiver counselling \\
\hline & Caregiver counselling & & \\
\hline Wheelchair management & Self-care activities & Intervention in an accident & Assistive devices \\
\hline Wheelchair assessment & Activity adaptation & Education & Needs assessment \\
\hline Wheelchair adaptation & Energy conservation & Practical skills training & Unmet needs: Advice \\
\hline Wheelchair skills training & Practical skills training & Caregiver counselling & Selection and aims \\
\hline Level driving & Dressing & & Costs \\
\hline Turns & Toileting & & Installation and use \\
\hline Driving in reverse & Bathing/showering & & Practical training \\
\hline Slope & Grooming & & Caregiver counselling \\
\hline Kerbs and obstacles & Feeding & & \\
\hline Other skills & Caregiver counselling & & \\
\hline
\end{tabular}

ADL: basic activities of daily living.

\section{Instruments and data collection}

The participants were assessed on admission to the ADL intervention (pre-intervention T1, baseline) and after completion of this intervention (post-intervention T2). Assessments were carried out by one of the authors (LS). Demographic data at baseline were recorded: age, gender, education level, marital status, children, and living alone before amputation. Clinical data of amputation were extracted from the medical records: level, cause (vascular vs. other), and time to ADL intervention (days), that is, time from amputation until the first day of the ADL intervention. The use of assistive devices for mobility was assessed at baseline, and this variable was dichotomized into the following categories: wheelchair (only) vs. use of walking aids (e.g., crutches). The duration of the ADL intervention was assessed (days). The destination of participants was determined at T2: discharge from rehabilitation vs. the continuation in the rehabilitation program (physiotherapy), either as inpatient or as outpatient.

Functional independence in basic ADL was the primary outcome, and this variable was measured by the BI. The BI is a 10-item ordinal scale that measures independence in the domains of self-care and mobility. It was developed as a measure to assess the degree of personal assistance required to complete $10 \mathrm{ADL}$ in patients receiving rehabilitation for neuromuscular and musculoskeletal conditions. [41] These 
activities are scored in steps of five points on a scale ranging from dependence to total independence. Bathing and grooming are scored 0 or 5; feeding, dressing, bladder control, bowel control, toileting, and stairs are scored 0, 5 or 10; bed-chair transfers and walking are scored 0, 5, 10 or 15 . The 10 items are summed, and a total score ranging from 0 (total dependence) to 100 (complete independence) is obtained. This study used the BI due to lack of specific scales to analyze self-care activities in lower-limb amputees. Moreover, the BI has been used in the ADL evaluation of lower-limb amputees previously.[33,34,36,38,42-45] A systematic review [46] examined the metric properties and clinical utility of the instruments for measuring rehabilitation outcomes of lower-limb amputees and concluded that the BI has good reliability and adequate validity for the amputee population; regarding responsiveness, although one study of an amputee population showed adequate results,[47] this review recommended further research.

The BI was administered twice in the rehabilitation service, once at baseline and once at T2. Changes in functional independence were assessed by comparing the scores on the BI at baseline with measurements taken at T2: total score and the scores on each of the six self-care activities addressed by this ADL intervention.

The secondary outcome measure assessed changes in the presence of unmet needs for home adaptation in the group of participants who were living in their homes before amputation. In this study, there was an unmet need when the occupational therapist identified that an adaptation (assistive device or removal of an architectural barrier) was necessary for basic ADL, but this adaptation was not available at the participant's home. Home was divided into three areas: entrance, bathroom, and other internal areas; in each of these areas, the presence of an unmet need for home adaptation was determined at T1 and T2 (yes/no). Moreover, home adaptations made between T1 and T2 were evaluated.

\section{Analysis}

Statistical analyses were performed using SPSS version 19 for Windows (Armonk, NY). The Kolmogorov-Smirnov test was used to determine the normal distribution. Age was the only quantitative variable that followed a normal distribution. Participants were divided into two groups at T1 (inpatient vs. outpatient). Baseline characteristics (demographics, clinical data, assistive devices for mobility, and the total score on the $\mathrm{BI}$ ) and the duration of the ADL intervention were compared between these two groups, using the Mann-Whitney $U$-test for continuous variables and chi-square test or Fisher's exact test for categorical variables.

The Wilcoxon test was used to assess BI scores' changes between baseline and T2. The effect size $(r)$ [ES $(r)]$ was calculated by dividing the $Z$ statistic of the Wilcoxon tests by the square root of the total number of observations;[48] an ES( $r)$ of 0.10 constitutes a small effect, 0.30 a medium effect and 0.50 a large effect.[49] We used a paired $t$-test to assess changes in the number of self-care activities performed independently (without personal assistance) between $\mathrm{T} 1$ and $\mathrm{T} 2$, according to the $\mathrm{BI}$; in the group of participants with bilateral amputation, this analysis was performed using the Wilcoxon signed-rank test. Mc Nemar's test was used to assess changes in the presence of unmet needs for home adaptation between baseline and T2. Level of significance was set at a $p$ values of $<0.05$.

A multivariate logistic regression analysis was performed to identify variables associated with a successful intervention (successful vs. unsuccessful); independent variables included demographics, clinical data, and ADL intervention characteristics. Level of amputation was dichotomized (unilateral transtibial vs. another category). First, the association of each independent variable with a successful intervention was assessed in univariate analyses using $t$-tests or the Mann-Whitney $U$-test for continuous variables and chi-square tests for categorical variables. All variables with a $p$ values $<0.05$ were then entered into a multivariate logistic regression analysis; through backward stepwise elimination, all noncontributing variables $(p>0.05)$ were excluded, leading to the best-fit model. Odds ratios (OR) with corresponding $95 \%$ confidence intervals (CI) were calculated for each of the contributing factors. The Cox-Snell R-squared value was calculated. A Hosmer-Lemeshow test of the goodness of fit was performed. 


\section{Results}

Descriptive characteristics of the 52 participants at baseline are described in Table 2. The most frequent amputation was unilateral transfemoral $(65.4 \%)$. The cause of amputation for the majority of participants was vascular $(86.5 \%)$. More than $90 \%$ of participants used only a wheelchair for mobility at baseline (90.4\%). The time from amputation to admission for the ADL intervention was 16 days [median, interquartile range (IQR) 21.5]. The duration of the ADL intervention was 9 days (median, IQR 5.8). After completing this intervention, 39 participants (75\%) continued with the rehabilitation program (physiotherapy), and 13 (25\%) finished the rehabilitation. Ten patients were discharged home, and three were discharged to a long-term residential facility.

Table 2. Descriptive characteristics of subjects at baseline $(n=52)$.

\begin{tabular}{lr}
\hline Sample characteristics & Value $-n(\%)$ \\
\hline & \\
Demographic characteristics & $65.5(11.8)$ \\
Age in years, mean (SD) & $42(80.8)$ \\
Male & \\
Level of education & $40(76.9)$ \\
$\quad$ Lower (<10 years) & $12(23.1)$ \\
$\quad$ Higher ( $\geq 10$ years) & $29(55.8)$ \\
Married & $40(76.9)$ \\
With children & $12(23.1)$ \\
Living alone before amputation & \\
Amputation & $12(23.1)$ \\
Level of amputation & $34(65.4)$ \\
$\quad$ Lower (transtibial) & $1(1.9)$ \\
$\quad$ Transgenual or transfemoral & $5(9.6)$ \\
$\quad$ Disarticulation hip & \\
$\quad$ Bilateral & $45(86.5)$ \\
Cause & $7(13.5)$ \\
$\quad$ Vascular (e.g., diabetes mellitus) & $16.5(21.5)$ \\
$\quad$ Other (e.g., trauma, neoplasm) & \\
Time from amputation to ADL intervention, median (IQR) & \\
Assistive devices for mobility & $47(90.4)$ \\
Wheelchair (only) & $45(86.5)$ \\
Walking aid (e.g., crutches) & \\
Inpatient & \\
\hline
\end{tabular}

SD: standard deviation; ADL: basic activities of daily living; IQR: interquartile range.

Forty-five participants were inpatients (inpatient group), and seven participants were outpatients (outpatient group) at T1. The baseline characteristics of these two groups were compared. Significant differences in educational level and marital status were found; the outpatient group had a higher educational level $(p=0.005)$, and the percentage of married individuals was higher than in the inpatient group $(p=0.013)$. However, there were no significant differences in other baseline characteristics. With regard to the duration of the ADL intervention, there was no significant difference between these two groups. 


\section{Effects on functional independence}

There was a significant change in BI scores from $\mathrm{T} 1$ to $\mathrm{T} 2$ assessments: the total score was 60 at baseline (median, range 30-80, IQR 22.5), and this total score was 80 (median, range 40-95, IQR 10) at T2 $(p<0.001)$. Regarding the six self-care activities addressed by this ADL intervention, Table 3 shows changes in BI scores between baseline and T2. The Wilcoxon test showed a statistically significant improvement in functional independence in five of the six activities studied; in four of these activities, $\mathrm{ES}(r)$ was greater than 0.5 ; in feeding, $\mathrm{ES}(r)$ was small. In grooming, the improvement of independence was not significant. In bilateral amputees $(n=5)$, the percentages of independent performance at T2 in dressing, transfers, toileting, and bathing/showering (according to the BI) were 100, 80, 60, and 40\%, respectively.

Table 3. Functional independence in ADL, before and after intervention $(n=52)$.

\begin{tabular}{|c|c|c|c|c|c|c|}
\hline & \multicolumn{2}{|c|}{ Pre-intervention (T1) } & \multicolumn{2}{|c|}{ Post-intervention (T2) } & \multirow[b]{2}{*}{$p$ values } & \multirow[b]{2}{*}{ Effect size } \\
\hline & Independence $(\%)^{\mathrm{a}}$ & Median $(\mathrm{IQR})^{\mathrm{a}}$ & Independence $(\%)^{\mathrm{a}}$ & Median (IQR) ${ }^{\mathrm{a}}$ & & \\
\hline Bathing/showering & 9.6 & $0(0)$ & 61.5 & $5(5)$ & $<0.001^{\mathrm{b}}$ & 0.51 \\
\hline Toileting & 11.5 & $5(0)$ & 86.5 & $10(0)$ & $<0.001^{\mathrm{b}}$ & 0.63 \\
\hline Bed-chair transfers & 17.3 & $10(5)$ & 88.5 & $15(0)$ & $<0.001^{\mathrm{b}}$ & 0.57 \\
\hline Dressing & 28.8 & $5(5)$ & 98.1 & $10(0)$ & $<0.001^{\mathrm{b}}$ & 0.55 \\
\hline Feeding & 90.4 & $10(0)$ & 100 & $10(0)$ & $0.025^{\mathrm{b}}$ & 0.22 \\
\hline Grooming & 94.2 & $5(0)$ & 100 & $5(0)$ & 0.083 & - \\
\hline
\end{tabular}

ADL: basic activities of daily living; IQR: interquartile range.

${ }^{\text {a }}$ Based on the Barthel Index.

${ }^{\mathrm{b}}$ Indicates significant finding $(p<0.05)$.

The number of self-care activities performed independently increased significantly between $\mathrm{T} 1$ and $\mathrm{T} 2$, according to the $\mathrm{BI}$; the number of activities without personal assistance was 2 at baseline (median, range $1-5$, IQR 1); this number was 6 at T2 (median, range 2-6, IQR 1) $(p<0.001)$. This improvement was also statistically significant in the group of participants with bilateral amputation $(n=5)$ : the number of activities carried out without personal assistance was 2 at baseline (median, range 1-3, IQR 1). This number was 5 at T2 (median, range 3-6, IQR 2.5) $(p=0.042)$.

\section{Effects on home adaptation needs}

Table 4 presents the home adaptations for basic ADL made during this intervention, in the group of participants who were living in their homes before amputation $(n=48)$. The adaptations made more frequently were the installation of grab bars for bathroom and shower seats. 
Table 4. Home adaptations made during the ADL intervention $(n=48)$.

\begin{tabular}{lc}
\hline Home adaptations for ADL & $\begin{array}{c}\text { Between T1 and T2: } \\
\text { participant made the adaptation }\end{array}$ \\
\hline Removal of architectural barriers & \\
Install a ramp/remove steps & 12.5 \\
Install a lift/stairlift & 0 \\
Increase width of doors & 6.3 \\
Install a shower & 8.3 \\
Use of an assistive device & \\
Raised toilet seat & 12.5 \\
Toilet grab rail/grab bar & 29.2 \\
Bath/shower grab bar & 33.3 \\
Bathtub seat (e.g., swivel-seat) & 4.2 \\
Bath board & 0 \\
Shower seat & 27.1 \\
\end{tabular}

ADL: basic activities of daily living; T1: baseline; T2: post-intervention. Data are presented as \%.

Regarding the group of participants who lived in their homes before amputation $(n=48)$, Table 5 details the presence of unmet needs for home adaptation, before and after intervention, in the three home areas studied (entrance, bathroom, and other internal areas). The proportion of homes with an unmet need for adaptation decreased significantly within the home between $\mathrm{T} 1$ and $\mathrm{T} 2$; no significant differences were found at the entrance. Unmet needs for adaptation were more frequent in bathroom at baseline and after the ADL intervention.

Table 5. Unmet needs for home adaptation, before and after intervention $(n=48)$.

\begin{tabular}{lccc}
\hline & Pre-intervention (T1) Post-intervention (T2) $p$ values \\
\hline & & & \\
Unmet need for home adaptation & & 77.1 & 0.125 \\
Entrance & 95.4 & 79.2 & $0.008^{\mathrm{a}}$ \\
Bathroom & 54.2 & 41.7 & $0.031^{\mathrm{a}}$ \\
Other internal areas & & & \\
\hline
\end{tabular}

Data are presented as $\%$.

${ }^{\mathrm{a}}$ Indicates significant finding $(p<0.05)$. 


\section{Factors associated with a successful intervention}

Thirty-two participants (61.5\%) performed without personal assistance all six ADL studied at T2 (successful intervention). Univariate analysis found that the successful intervention group had lower age $(p<0.001)$, a higher rate of amputations due to vascular cause $(p=0.03)$ and received an intervention of longer duration $(p=0.04)$. In the multivariate model $(n=52)$, only age was significantly associated with a successful intervention $[p<0.001$; OR $0.66(95 \%$ CI $0.52-0.83)]$. The Cox-Snell $R$-squared value was 0.60 . A Hosmer-Lemeshow test produced a chi-square value of $9.09(p=0.34)$, suggesting that the model had a good explanatory power.

\section{Discussion}

The primary goal of this study was to evaluate the influence of a pre-prosthetic ADL intervention on independence in self-care activities. No studies were found that systematically evaluated the impact of ADL programs for people with lower-limb amputation at the pre-prosthetic phase. In a sample characterized by an advanced mean age, a very recent amputation and wheelchair use, our findings suggested a significant and rapid functional recovery in self-care. Despite the fact that ADL were seriously compromised after amputation, post-intervention BI values were significantly higher than those observed at baseline in five of the six activities studied. In bathing and showering, toileting, bed-chair transfers and dressing, the impact on functional independence was large. Regarding upper limb activities, this study showed a significant improvement in feeding (unlike grooming) due to the implementation of training strategies with adaptations and assistive devices aimed to compensate for limitations of the cutting-food task, produced by conditions such as osteoarthritis or traumatic hand injuries. Our results therefore suggested that pre-prosthetic ADL training has a clinically meaningful effect on functional independence. After completing this intervention, only a quarter of the participants were discharged from rehabilitation; early onset and short duration of this ADL intervention argue this fact. Most participants continued with physiotherapy, a program lasting $\sim 6-10$ weeks at the pre-prosthetic phase, according to previous literature.[14,18]

The main contribution of our study was to examine the impact of a short intervention at an early stage, applied within the first weeks after amputation, in contrast to the scarce literature on this topic, characterized by assessing changes in functional independence between admission and discharge of a rehabilitation program. It is difficult to compare the results with previous research due to considerable differences in the interventions implemented and the settings, the heterogeneity of study populations and the diversity of outcome measures. Regarding the findings on the BI scores, functional independence after this ADL intervention was higher than the post-rehabilitation BI score reported by Chen et al. [33] recently in an inpatient rehabilitation setting (69 points vs. 80 in our study). Our results were similar to the functional improvement found in a sample of transfemoral amputees who completed prosthetic rehabilitation; [36] the interval between amputation and admission to rehabilitation was 67 days (median), and the BI score increased from 62.9 (admission) to 88 points (discharge); however, the length of this rehabilitation stay (median 89 days) was much greater than the duration of the ADL intervention. The post-intervention BI score of the current study was consistent with the functional level found in a prospective study;[45] most participants of this follow-up study were admitted to prosthetic training. One year after amputation, the mean BI score was 79 points (total sample); however, this finding was substantially lower in the subgroup of non-walking patients (mean BI: 56 points). With respect to nonprosthetic rehabilitation, this ADL intervention achieved greater functional independence than postrehabilitation BI outcomes of a study with frail elderly;[34] moreover, the rehabilitation stay was large (median 142 days).

Recently, a multidisciplinary rehabilitation program improved functional independence in geriatric patients, assessed by the functional independence measure (FIM);[12] however, participants with prosthetic mobility obtained a lower degree of independence at the end of the rehabilitation process (median stay: 96 days) than that achieved by the sample of this pre-prosthetic study. Differences in the age of the samples may contribute to the dissimilarity between the two studies (74 years vs. 65 in our study). Panesar et al. [35] also used the FIM instrument for evaluating an inpatient rehabilitation program 
for vascular amputees; more than $88 \%$ of participants received prosthesis at discharge. This study found that independence in basic ADL increased significantly after rehabilitation; however, in toileting and bathing activities, the findings at rehabilitation discharge reflected the requirement of assistive devices, the presence of safety risks and/or the need of a greater duration than a reasonable time to perform these activities. Finally, the results of the current study contrast with those obtained in a research with people with vascular transtibial amputation without prosthesis;[50] only $22 \%$ of participants were independent in the six basic ADL assessed by the Katz Index at hospital discharge.

In this ADL intervention, participants regained their best possible independence in basic activities by the learning of non-prosthetic mobility skills. Previous studies found that a high proportion of amputees are not candidates for prosthetic rehabilitation, $[2,22-28]$ so that wheelchair use is not only the main means of mobilization at the pre-prosthetic phase,[18] but these wheelchair skills would be essential for autonomy of many participants after rehabilitation. Accordingly, the literature has suggested that an early program of transfers and wheelchair skills is an appropriate goal for most amputees.[15,18,20,24] The candidates for prosthetic rehabilitation may also benefit from this early and pre-prosthetic ADL intervention for several reasons. First, the use of prosthesis after rehabilitation is usually limited to part of the day [19,51] and, consequently, many participants will use the non-prosthetic mobility skills learned in this intervention at various times of their daily lives. In addition, there is a high incidence of contralateral amputations and reamputations.[10,13,52] The ADL training encourages learning new and effective strategies aimed to promote the return to patients' basic activities; consequently, this intervention may increase self-confidence, locus of control and motivation [14,15,18] because it shows the participants their potential for autonomy during a phase characterized by deconditioning, dependence and emotional disorders. Moreover, this intervention can contribute to the maintenance of basic functions for achieving prosthetic fitting such as postural control, strength, and physical fitness by strategies such as balance training, transfers and wheelchair self-propulsion.[19,39] Safety education, another component of this ADL intervention, is an issue of particular relevance due to the high prevalence of falls during the first weeks after amputation, $[18,53,54]$ and injuries can be an obstacle for the prosthetic fitting. $[19,21]$

Little is known about the predictors of ADL outcomes after a lower-limb amputation. In this study, we explored participant factors and intervention characteristics as possible determinants of the effectiveness of this ADL intervention. Age was an important predictor of functional recovery. A younger age was significantly associated with the independent performance of all self-care activities after this intervention, which is consistent with previous studies.[36,55,56] Therefore, age should be a factor to consider when rehabilitation professionals advise patients and caregivers about the expected results in basic ADL. Level of amputation was not a significant predictor for a successful intervention, despite the fact that this factor was significantly associated with walking ability in the literature.[24,39] In line with previous studies,[34,55,57] the findings showed the potential of people with bilateral or transfemoral amputation regarding independence in ADL and suggested that an optimal functional recovery is a realistic goal for a large proportion of this population regardless of the level of amputation.

Home adaptation was a key strategy in this study, given the critical role of person-environment interactions. The biopsychosocial model of functioning and disability [58] has emphasized the importance of contextual factors in daily activities and disability. In people with lower-limb amputation, assistive devices, and home adaptations facilitate the return home;[59] improve the performance of self-care, domestic and social activities;[42] and reduce injuries.[53] In the previous research, information on the presence of obstacles to daily functioning at homes is very limited. This study showed the high presence of environmental barriers. The bathroom was the least accessible space and, in turn, the place where most adaptations were implemented, consistent with previous studies.[6,51] Adaptations such as grab bars, seats, and raised toilets promote balance, energy conservation, and comfort, with an affordable cost for most participants. After this intervention, significant improvements were recorded in the coverage of home adaptation needs. The participation in a pre-prosthetic program of education and training in home adaptations had a positive effect on accessibility in the home, an essential facilitator for autonomy and safety. 
Some limitations warrant further consideration. The main limitation was the lack of a control group. Accordingly, the changes that occurred during the study cannot be attributed to the effects of intervention alone. In addition, the sample size was relatively small. The findings of the multivariate analysis represent small subgroups and, therefore, the results need to be interpreted with caution. The study population consisted of patients with ADL limitations and potential for improvement of functional independence, referred to a single rehabilitation service at the pre-prosthetic phase, thus making it difficult to generalize the results to all Spanish rehabilitation settings and for all amputees at this stage. Moreover, we did not take into account some potentially important measurements such as comorbidity or cognitive status due to limitations in the collection of this information in the medical records of some participants. Finally, this study did not include a post-intervention follow-up. Although the gains in functional independence were substantial, follow-up assessments are required to determine the extent to which the gains are maintained.

\section{Conclusion}

This research on the impact of an ADL intervention at the pre-prosthetic phase has not been undertaken previously, making this study unique. It is hoped that this study will help to form the basis for future research on autonomy and daily activities in this population. The findings showed that an early and short-term ADL intervention in a sub-acute rehabilitation setting is an effective method for functional recovery. Participation in this program led to significant improvements in the ability to perform basic ADL, and the effects on independence were large in most self-care activities analyzed. Our study showed that age was a determinant of functional outcomes, and most subjects achieved independence regardless of the level of amputation. The intervention also highlighted the importance of advice about adaptations to achieve an accessible home, which facilitates the performance of daily tasks. This ADL program may represent an efficient use of scarce health care resources for this growing population group, given the optimal functional results obtained and the short duration of practical training. The findings suggest that pre-prosthetic ADL programs should be included in the rehabilitation strategies for lower-limb amputees with the aim of promoting autonomy and safety in daily activities.

\section{Disclosure statement}

The authors report no declarations of interest.

\section{References}

[1] Dillingham TR, Pezzin LE, MacKenzie EJ. Limb amputation and limb deficiency: epidemiology and recent trends in the United States. South Med J. 2002;95:875-883.

[2] Fletcher DD, Andrews KL, Butters MA, et al. Rehabilitation of the geriatric vascular amputee patient: a population-based study. Arch Phys Med Rehabil. 2001;82:776-779.

[3] Fletcher DD, Andrews KL, Hallett JW, Jr, et al. Trends in rehabilitation after amputation for geriatric patients with vascular disease: implications for future health resource allocation. Arch Phys Med Rehabil. 2002;83:1389-1393.

[4] Varma P, Stineman MG, Dillingham TR. Epidemiology of limb loss. Phys Med Rehabil Clin N Am. 2014;25:1-8.

[5] Ziegler-Graham K, MacKenzie EJ, Ephraim PL, et al. Estimating the prevalence of limb loss in the United States: 2005 to 2050. Arch Phys Med Rehabil. 2008;89:422-429.

[6] Durović A, Ilić D, Brdareski Z, et al. Pain, functional status, social function and conditions of habitation in elderly unilateraly lower limb amputees. Vojnosanit Pregl. 2007;64:837-843.

[7] Holzer LA, Sevelda F, Fraberger G, et al. Body image and self-esteem in lower-limb amputees. PLoS One. 2014;9:e92943.

[8] Geertzen J, van der Linde H, Rosenbrand K, et al. Dutch evidence-based guidelines for amputation and prosthetics of the lower extremity: amputation surgery and postoperative management. Part 1 . Prosthet Orthot Int. 2015;39:351-360.

[9] McKechnie PS, John A. Anxiety and depression following traumatic limb amputation: a systematic review. Injury. 2014;45:1859-1866. 
[10] Dillingham TR, Pezzin LE, Shore AD. Reamputation, mortality, and health care costs among persons with dysvascular lower-limb amputations. Arch Phys Med Rehabil. 2005;86:480-486.

[11] Dillingham TR, Pezzin LE. Rehabilitation setting and associated mortality and medical stability among persons with amputations. Arch Phys Med Rehabil. 2008;89:1038-1045.

[12] Hershkovitz A, Dudkiewicz I, Brill S. Rehabilitation outcome of post-acute lower limb geriatric amputees. Disabil Rehabil. 2013;35:221-227.

[13] Lim TS, Finlayson A, Thorpe JM, et al. Outcomes of a contemporary amputation series. ANZ J Surg. 2006;76:300-305.

[14] Coletta EM. Care of the elderly patient with lower extremity amputation. J Am Board Fam Pract. 2000;13:23-34.

[15] Esquenazi A, DiGiacomo R. Rehabilitation after amputation. J Am Podiatr Med Assoc. 2001;91:13-22.

[16] Geertzen J, van der Linde H, Rosenbrand K, et al. Dutch evidence-based guidelines for amputation and prosthetics of the lower extremity: rehabilitation process and prosthetics. Part 2. Prosthet Orthot Int. 2015;39:361-371.

[17] Meier RH, Heckman JT. Principles of contemporary amputation rehabilitation in the United States, 2013. Phys Med Rehabil Clin N Am. 2014;25:29-33.

[18] Robinson V, Sansam K, Hirst L, et al. Major lower limb amputation: what, why and how to achieve the best results. Orthop Trauma. 2010;24:276-285.

[19] Carnegie F. Traumatic amputation: management and occupational therapy. In: Mooney M, Ireson C, editors. Occupational therapy in orthopaedics and trauma. Chichester, UK: Wiley-Blackwell; 2009. p. 255-278.

[20] Meier RH, Melton D. Ideal functional outcomes for amputation levels. Phys Med Rehabil Clin N Am. 2014;25:199-212.

[21] Klarich J, Brueckner I. Amputee rehabilitation and preprosthetic care. Phys Med Rehabil Clin N Am. 2014;25:75-91.

[22] Batten HR, Kuys SS, McPhail SM, et al. Demographics and discharge outcomes of dysvascular and non-vascular lower limb amputees at a subacute rehabilitation unit: a 7-year series. Aust Health Rev. 2015;39:76-84.

[23] Davies B, Datta D. Mobility outcome following unilateral lower limb amputation. Prosthet Orthot Int. 2003;27:186-190.

[24] Fleury AM, Salih SA, Peel NM. Rehabilitation of the older vascular amputee: a review of the literature. Geriatr Gerontol Int. 2013;13:264-273.

[25] Fortington LV, Rommers GM, Geertzen JHB, et al. Mobility in elderly people with a lower limb amputation: a systematic review. J Am Med Dir Assoc. 2012;13:319-325.

[26] Nehler MR, Coll JR, Hiatt WR, et al. Functional outcome in a contemporary series of major lower extremity amputations. J Vasc Surg. 2003;38:7-14.

[27] Roth EV, Pezzin LE, McGinley EL, et al. Prosthesis use and satisfaction among persons with dysvascular lower limb amputations across postacute care discharge settings. PM\&R. 2014;6:11281136.

[28] Wu J, Chan TS, Bowring G. Functional outcomes of major lower limb amputation 1994-2006: a modern series. J Prosthet Orthot. 2010;22:152-156.

[29] Czerniecki JM, Turner AP, Williams RM, et al. The effect of rehabilitation in a comprehensive inpatient rehabilitation unit on mobility outcome after dysvascular lower extremity amputation. Arch Phys Med Rehabil. 2012;93:1384-1391.

[30] Pezzin LE, Padalik SE, Dillingham TR. Effect of postacute rehabilitation setting on mental and emotional health among persons with dysvascular amputations. PM\&R. 2013;5:583-590.

[31] Singh R, Hunter J, Philip A. The rapid resolution of depression and anxiety symptoms after lower limb amputation. Clin Rehabil. 2007;21:754-759.

[32] Stineman MG, Kwong PL, Kurichi JE, et al. The effectiveness of inpatient rehabilitation in the acute postoperative phase of care after transtibial or transfemoral amputation: study of an integrated health care delivery system. Arch Phys Med Rehabil. 2008;89:1863-1872.

[33] Chen C, Naidoo N, Er B, et al. Factors associated with nursing home placement of all patients admitted for inpatient rehabilitation in Singapore community hospitals from 1996 to 2005: a disease stratified analysis. PLoS One. 2013;8:e82697.

[34] Eijk MSV, Van Der Linde H, Buijck BI, et al. Geriatric rehabilitation of lower limb amputees: a multicenter study. Disabil Rehabil. 2012;34:145-150.

[35] Panesar BS, Morrison P, Hunter J. A comparison of three measures of progress in early lower limb amputee rehabilitation. Clin Rehabil. 2001;15:157-171.

[36] Traballesi M, Brunelli S, Pratesi L, et al. Prognostic factors in rehabilitation of above knee amputees for vascular diseases. Disabil Rehabil. 1998;20:380-384.

[37] Stineman MG, Kurichi JE, Kwong PL, et al. Survival analysis in amputees based on physical independence grade achievement. Arch Surg. 2009;144:543-551. 
[38] Bilodeau S, Hébert R, Desrosiers J. Lower limb prosthesis utilisation by elderly amputees. Prosthet Orthot Int. 2000;24:126-132.

[39] Sansam K, Neumann V, O'Connor R, et al. Predicting walking ability following lower limb amputation: a systematic review of the literature. J Rehabil Med. 2009;41:593-603.

[40] Zidarov D, Swaine B, Gauthier-Gagnon C. Quality of life of persons with lower-limb amputation during rehabilitation and at 3-month follow-up. Arch Phys Med Rehabil. 2009;90:634-645.

[41] Mahoney FI, Barthel DW. Functional evaluation: the Barthel index. Md State Med J. 1965;14:61-65.

[42] Collin C, Wade DT, Cochrane GM. Functional outcome of lowe limb amputees with peripheral vascular disease. Clin Rehabil. 1992;6:13-21.

[43] Datta D, Ariyaratnam R, Hilton S. Timed walking test: an all-embracing outcome measure for lowerlimb amputees? Clin Rehabil. 1996;10:227-232.

[44] O'Toole DM, Goldberg RT, Ryan B. Functional changes in vascular amputee patients: evaluation by Barthel index, PULSES profile and ESCROW scale. Arch Phys Med Rehabil. 1985;66:508-511.

[45] Pernot HFM, Winnubst GMM, Cluitmans JJM, et al. Amputees in Limburg: incidence, morbidity and mortality, prosthetic supply, care utilisation and functional level after one year. Prosthet Orthot Int. 2000;24:90-96.

[46] Deathe AB, Wolfe DL, Devlin M, et al. Selection of outcome measures in lower extremity amputation rehabilitation: ICF activities. Disabil Rehabil. 2009;31:1455.

[47] Traballesi M, Paolucci S, Lubich S, et al. Non-traumatic above-knee amputation in elderly patients. Results of rehabilitation and prognostic factors. Eura Medicophys. 1995;31:21-26.

[48] Field A. Discovering statistics using SPSS. 3rd ed. London, UK: Sage Publications Ltd; 2009.

[49] Cohen J. Statistical power analysis for the behavioral sciences. Hillsdale, MI: Lawrence Erlbaum Associates; 1988.

[50] Hermodsson Y, Ekdahl C. Assessing functional ability in patients with unilateral trans-tibial amputation for vascular disease. Scand J Occup Ther. 1998;5:167-172.

[51] Meatherall BL, Garrett MR, Kaufert J, et al. Disability and quality of life in Canadian Aboriginal and non-Aboriginal diabetic lower-extremity amputees. Arch Phys Med Rehabil. 2005;86:1594-1602.

[52] Johannesson A, Larsson G-U, Ramstrand N, et al. Incidence of lower-limb amputation in the diabetic and nondiabetic general population: a 10-year population-based cohort study of initial unilateral and contralateral amputations and reamputations. Diabetes Care. 2009;32:275-280.

[53] Gooday HMK, Hunter J. Preventing falls and stump injuries in lower limb amputees during inpatient rehabilitation: completion of the audit cycle. Clin Rehabil. 2004;18:379-390.

[54] Pauley T, Devlin M, Heslin K. Falls sustained during inpatient rehabilitation after lower limb amputation: prevalence and predictors. Am J Phys Med Rehabil. 2006;85:521-535.

[55] Schoppen T, Boonstra A, Groothoff JW, et al. Physical, mental, and social predictors of functional outcome in unilateral lower-limb amputees. Arch Phys Med Rehabil. 2003;84:803-811.

[56] Shin JC, Kim EJ, Park CI, et al. Clinical features and outcomes following bilateral lower limb amputation in Korea. Prosthet Orthot Int. 2006;30:155-164.

[57] Paul R, Masilamani S, Dwyer AJ. Evaluation of rehabilitated bilateral lower limb amputees: an Indian study. Disabil Rehabil. 2012;34:1005-1009.

[58] World Health Organization. International classification of functioning, disability and health. Geneva, Switzerland: World Health Organization; 2001.

[59] Jones L, Hall M, Schuld W. Ability or disability? a study of the functional outcome of 65 consecutive lower limb amputees treated at the royal South Sydney hospital in 1988-1989. Disabil Rehabil. 1993;15:184-188. 